\title{
The Interrelation between Incompressible Strips and Quantized Hall Plateaus
}

\author{
AFIF SIDDIKI and ROLF R. GERHARDTS \\ Max-Planck-Institut für Festkörperforschung, \\ Heisenbergstrasse 1, D-70569, \\ Federal Republic of Germany \\ a.siddiki@fkf.mpg.de
}

(Received 23 June 2004)

\begin{abstract}
We study the current and charge distribution of a two dimensional electron gas under strong perpendicular magnetic fields within the linear response regime. We show within a self-consistent screening theory that incompressible strips with integer values of local Landau-level filling factor exist for finite intervals of the magnetic field strength $B$. Within an essentially local conductivity model, we find that the current density in these $B$ intervals is confined to the incompressible strips of vanishing local longitudinal resistivity. This leads to vanishing longitudinal and exactly quantized Hall resistance, and to a nice agreement of the calculated Hall potential profiles with the measured ones.

PACS numbers:
\end{abstract}




\section{INTRODUCTION}

Recent investigations [1] with a scanning force microscope produced evidence, that the spatial distribution of the dissipative current, carried by a two-dimensional electron gas (2D EG) in a Hall bar under the conditions of the quantized Hall effect (QHE), is dominated by the existence of incompressible strips (ISs), which are expected to develop in the space regions where an integer number of Landau levels (LLs) is filled. 2] Subsequent theoretical work, 3. which calculated electron density and electrostatic potential within a self-consistent Thomas-Fermi-Poisson approximation, and current density and position-dependent electrochemical potential from a local version of Ohm's law, confirmed the experimental evidence and found that the current flows in the ISs, in which the local longitudinal resistivity vanishes. Due to the Thomas-Fermi approximation (TFA), incompressible strips were found for all values $B$ of the magnetic field below a threshold $B_{c}$, at which the center of the sample becomes incompressible. [3] This leads to a vanishing total resistance for $B<B_{c}$, to a potential drop in several steps across ISs, which may coexist near a sample edge, and, for $B<B_{c}$, to the absence of $B$ intervals with linear variation of the Hall potential across the sample. These shortcomings are not in agreement with experiment 1] and require an improvement of the model. $[3]$

\section{IMPROVEMENTS OF THE GÜVEN-GERHARDTS MODEL}

We have recently improved on the approach of Güven and Gerhardts [3] in two essential respects 4]. First, replacing the TFA by a Hartree-type approximation, which takes the finite extent of wavefunctions into account, we demonstrate that the existence of incompressible

strips is restricted to finite $B$ intervals below $B_{c}[4]$. Second, we relax the assumption of a strictly local relation between conductivity and electron density, which leads to singular current density even along isolated lines of integer LL-filling factors, and we simulate nonlocal effects by a suitable averaging of the local conductivity tensor [4].

Following the previous work, [3, 5] we calculate density profile and effective confinement potential of the 2D EG in thermal equilibrium self-consistently. Assuming that all charges, including a homogeneous positive background and induced charges on gates, defining the 
Hall bar (in $|x|<d$ ), are concentrated on the plane $z=0$ with translation invariance in $y$ direction, we calculate the potential $V(x)$ from the electron density $n_{\mathrm{el}}(x)$ solving Poisson's equation under suitable boundary conditions. [2, 3, 5] Electrostatic self-consistency requires that the density of the spin-degenerate 2D EG is calculated from

$$
n_{\mathrm{el}}(x)=\frac{2}{2 \pi l^{2}} \int d X_{0} \sum_{n=0}^{\infty} \frac{\left|\phi_{n, X_{0}}(x)\right|^{2}}{\exp \left(\left[E_{n}\left(X_{0}\right)-\mu^{\star}\right] / k_{B} T\right)+1},
$$

where $l=\sqrt{\hbar / m \omega_{c}}$ is the magnetic length, $X_{0}$ a center coordinate, $\mu^{\star}$ the electrochemical potential. The energy eigenvalues $E_{n}\left(X_{0}\right)$ and eigenfunctions $\phi_{n, X_{0}}(x)$ should be calculated from the single-electron Schrödinger equation with the confinement potential $V(x)$. In the previous work one assumed $V(x)$ to vary slowly on the scale $l$ and evaluated Eq.(II) in the TFA, $E_{n}\left(X_{0}\right) \approx E_{n}+V\left(X_{0}\right),\left|\phi_{n, X_{0}}(x)\right|^{2} \approx \delta\left(x-X_{0}\right)$, neglecting the extent of the wavefunctions. 2, 3, 5] This yields flat ISs in the density profile with local filling factor
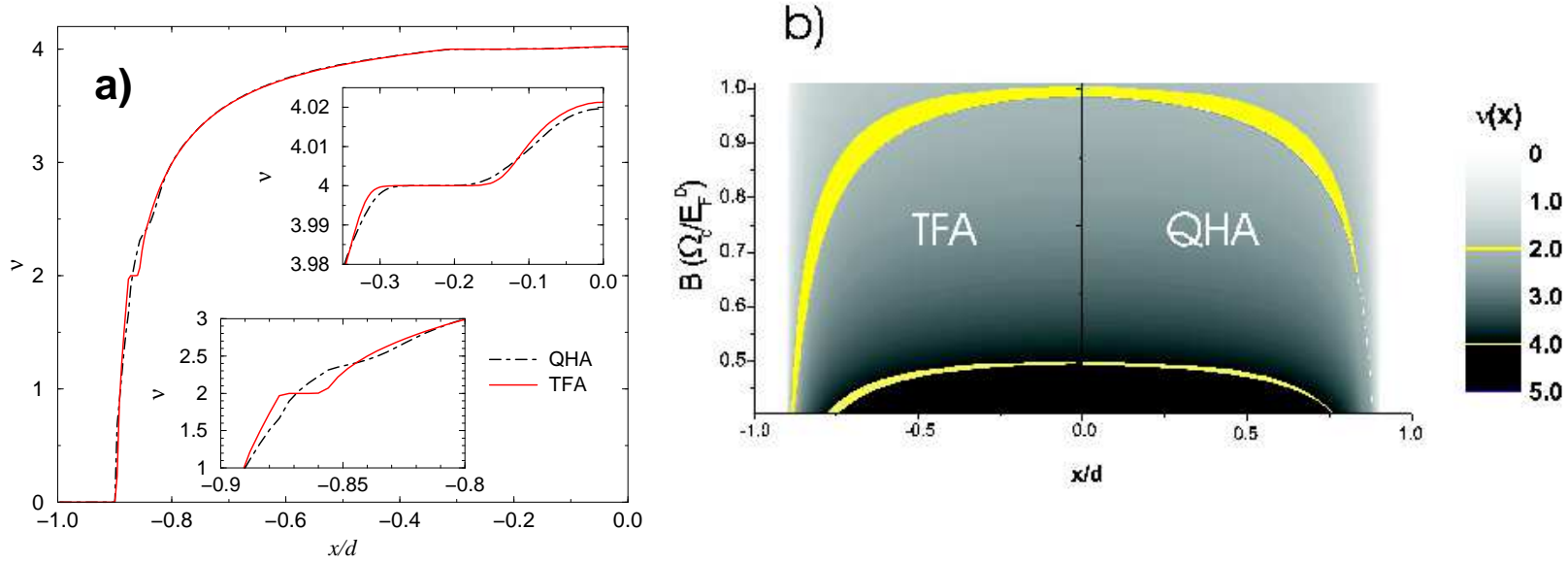

FIG. 1: (color online) (a) Calculated density profiles for the left half of a symmetric Hall bar. While the TFA yields incompressible strips with $\nu(x)=2$ and $\nu(x)=4$, only those with $\nu(x)=4$ survive in the QHA. (b) Local filling factors (gray scale plot) versus position $x$ and cyclotron energy $\Omega_{c} \equiv \hbar \omega_{c}$. ISs (light) exist in the TFA for all $B$ with $\Omega_{c} / E_{F}^{0}<1$, in the QHA, however, only for $1.0>\Omega_{c} / E_{F}^{0}>0.65$ and $0.5>\Omega_{c} / E_{F}^{0}>0.4 . d=3.1 \mu \mathrm{m}$, average electron density $3 \cdot 10^{11} \mathrm{~cm}^{-2}$, temperature $k_{B} T / E_{F}^{0}=0.02, E_{F}^{0}=n_{\mathrm{el}}^{0}(0) \pi \hbar^{2} / m$, where $n_{\mathrm{el}}^{0}(0)=n_{\mathrm{el}}(0)$ at $T=0, B=0$.

$\nu(x) \equiv 2 \pi l^{2} n_{\mathrm{el}}(x)=2$ for all magnetic-field values $B<B_{2}\left(\equiv B_{c}\right)$, with $\nu(x)=4$ for all $B<B_{4}\left(=B_{2} / 2\right)$, etc. This is, however, an artifact of the TFA. If one takes the width $\lambda_{W}$ of the wavefunctions into account, e.g. in the full Hartree approximation, one 
finds that the ISs calculated in the TFA are smeared out if their width becomes smaller than $\lambda_{W}$. This is already seen for the "quasi-Hartree approximation" (QHA), which uses again $E_{n}\left(X_{0}\right) \approx E_{n}+V\left(X_{0}\right)$ but replaces the $\phi_{n, X_{0}}(x)$ by the Landau wavefunctions of the unconfined 2D EG, as is demonstrated in Fig पa and in Fig \b, which shows within the QHA no ISs for $B_{4} \equiv 0.5 B_{2}<B<0.65 B_{2}$.

The stationary state with an applied dissipative current $I$ was described [3] by a local Ohm's law $\mathbf{E}(\mathbf{r})=\hat{\rho}(\mathbf{r}) \mathbf{j}(\mathbf{r})$, with $\hat{\rho}(\mathbf{r})=\left[\hat{\sigma}\left(n_{\mathrm{el}} \mathbf{r}\right)\right]^{-1}$, which allows us to calculate current density $\mathbf{j}(\mathbf{r})$ and driving field $\mathbf{E}(\mathbf{r})=\nabla \mu^{\star}(\mathbf{r}) / e$ with the constant components $j_{x} \equiv 0$ and $E_{y}(x) \equiv E_{y}^{0}$, and with $j_{y}(x)=E_{y}^{0} / \rho_{l}(x)$, and $E_{x}(x)=E_{y}^{0} \rho_{H}(x) / \rho_{l}(x)$, with $\rho_{l}(x)$ the longitudinal and $\rho_{H}(x)$ the Hall resistivity. This yields the Hall resistance $R_{H}=V_{H} / I$ and the longitudinal resistance $R_{l}=2 d E_{y}^{0} / I$ :

$$
I=E_{y}^{0} \int_{-d}^{d} d x 1 / \rho_{l}(x), \quad V_{H}=E_{y}^{0} \int_{-d}^{d} d x \rho_{H}(x) / \rho_{l}(x) .
$$

The feedback of the applied current on the (measurable) change of the electrostatic potential [1] was considered by requiring the stationary state to be in local equilibrium. [3] Here we consider only a linear response situation, in which the current-induced change of the electrostatic potential equals that of the electrochemical potential. To avoid spurious singularities of the integrals in Eq.(21) along isolated lines of vanishing $\rho_{l}(x)$, i.e. integer $\nu(x)$, which are an artifact of the strictly local model, we average the conductivity tensor, over a length $\lambda$ of the order of the mean electron distance (Fermi wavelength): $\hat{\bar{\sigma}}(x)=\frac{1}{2 \lambda} \int_{-\lambda}^{\lambda} d \xi \hat{\sigma}(x+\xi)$.

Starting with the self-consistent Born approximation [6] for the conductivity tensor of a homogeneous 2D EG, $\hat{\sigma}(\nu)$, replacing the filling factor $\nu$ by the local $\nu(x)$ calculated within the TFA, and performing the spatial average, we find strips with vanishing denominators in Eq.(2) only for ISs which are wider than $2 \lambda$ and survive also in the QHA. If such wide ISs with $\nu(x)=2 k$ exist in a certain $B$-interval, they carry all the current (cf. Fig 2 a) and lead, for $T \rightarrow 0$, to exactly quantized resistance values, $R_{H}=h /\left(e^{2} 2 k\right), R_{l}=0$. For all other $B$-values the current is spread over the sample, and Eq.(21) yields reasonable average values for $R_{H}$ and $R_{l}$, as is demonstrated in Fig 2 b. 

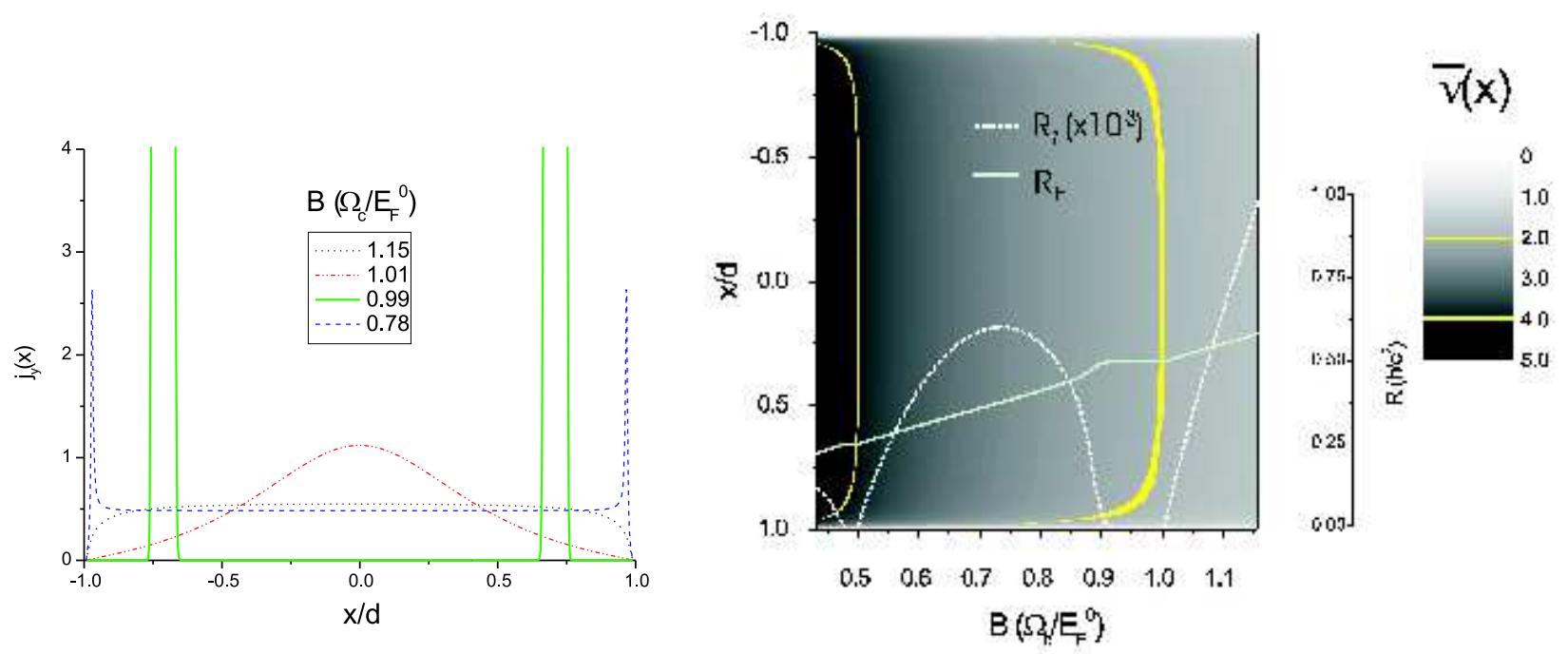

FIG. 2: (color online) (a) Current distribution for selected $B$ values. Only for $\Omega_{c} / E_{F}^{0}=0.99$ (solid line) the current is confined to the incompressible strips, which survived the averaging. $\lambda=20 \mathrm{~nm}$. (b) Gray scale plot of the averaged $\bar{\nu}(x) \approx\left(h / e^{2}\right) \bar{\sigma}_{H}(x)$ versus $B$ and $x$, together with Hall (solid) and longitudinal (broken light line) resistance; $d=7.5 \mu \mathrm{m}, \lambda=20 \mathrm{~nm}, k_{B} T / E_{F}^{0}=0.02$.

\section{SUMMARY}

In conclusion, the spatial averaging of the conductivity tensor, which simulates nonlocal effects and eliminates spurious singularities due to narrow ISs, which are an artifact of the TFA and do not survive in a Hartree-type approximation, represents an essential improvement of the theory and allows us to calculate reasonable results for the longitudinal $\left(R_{l}\right)$ and the Hall $\left(R_{H}\right)$ resistance as continuous functions of $B$, with high-precision plateau values in the $B$ intervals in which sufficiently wide ISs exist. Furthermore, we find three different types of the variation of the Hall potential across the sample (Fig [3), in excellent agreement with the experiments. 1]

\section{Acknowledgments}

We acknowledge useful discussions with E. Ahlswede and J. Weis, and support by the Deutsche Forschungsgemeinschaft, SP "Quanten-Hall-Systeme”, GE306/4-2.

[1] E. Ahlswede, P. Weitz, J. Weis, K. v. Klitzing, K. Eberl, Physica B 298, 562 (2001). 

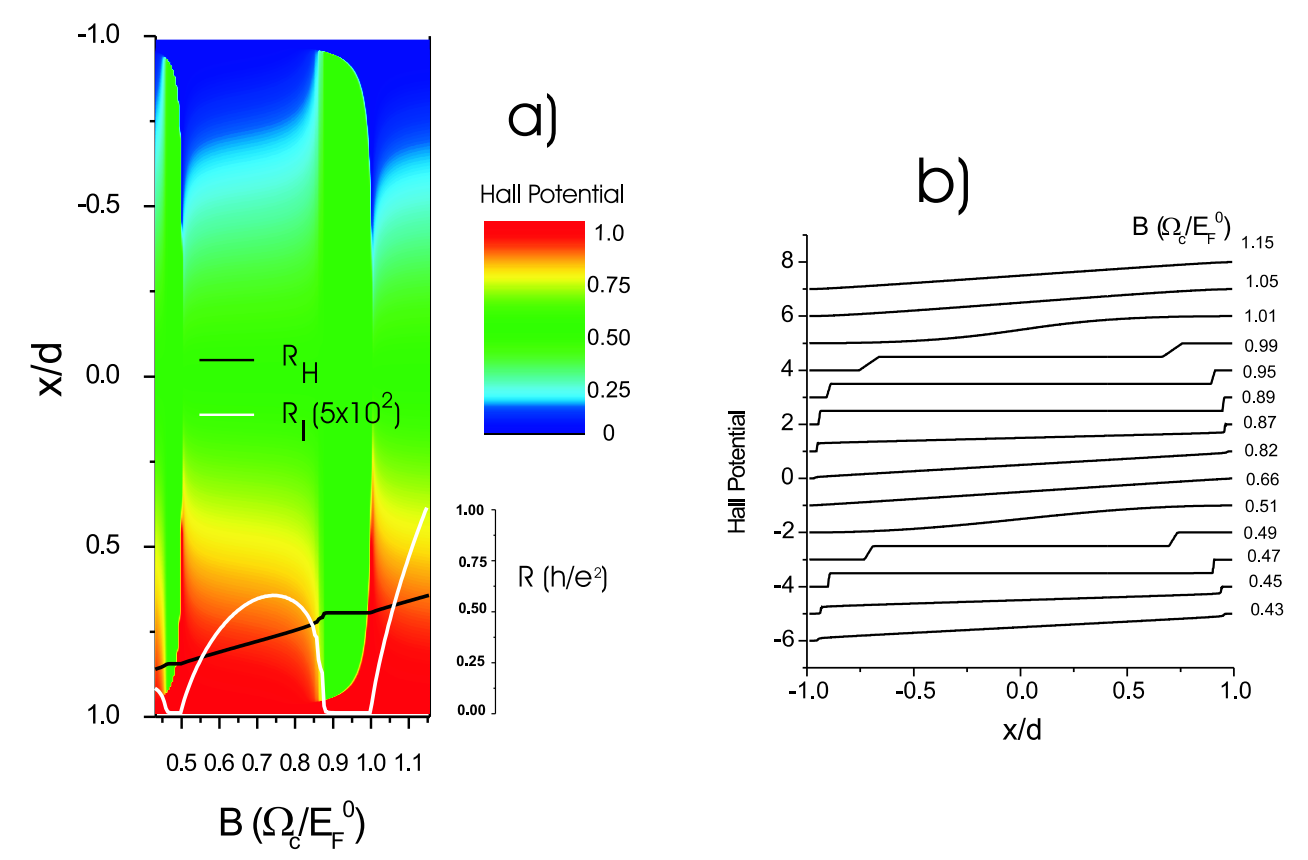

FIG. 3: (color online) (a) Plot of the scaled Hall potential $\Phi(x) / \Phi(d), \Phi(x)=\int_{-d}^{x} d \xi \bar{\sigma}_{H}(\xi) / \bar{\sigma}_{l}(\xi)$ versus $B$, together with $R_{H}(B)$ (black) and $R_{l}(B)$ (white line). $d=7.5 \mu \mathrm{m}, k T / E_{F}^{0}=0.0124$. (b) $\Phi(x) / \Phi(d)$ for selected values of $B$, vertically offset [cuts through (a)]. A stepwise variation is seen for $B$ in a plateau of the QHE, a linear variation if $B$ is far from a plateau, and a nonlinear variation in the center for $B$ near a plateau edge.

[2] D. B. Chklovskii, B. I. Shklovskii, and L. I. Glazman, Phys. Rev. B 46, 4026 (1992).

[3] K. Güven and R. R. Gerhardts, Phys. Rev. B 67, 115327 (2003).

[4] A. Siddiki and R. R. Gerhardts, cond-mat/0406199 (unpublished).

[5] J. H. Oh and R. R. Gerhardts, Phys. Rev. B 56, 13519 (1997).

[6] T. Ando, A. B. Fowler, and F. Stern, Rev. Mod. Phys. 54, 437 (1982). 